\title{
Validación de las Escalas de Inhibición Sexual/Excitación Sexual- Forma Breve (SIS/SES-SF)
}

\section{Validation of the Sexual Inhibition/Sexual Excitation Scales-Short Form (SIS/SES-SF)}

\author{
Nieves Moyano \\ Juan Carlos Sierra \\ Centro de Investigación Mente, Cerebro y Comportamiento (CIMCYC), Universidad de Granada, España
}

Rec (05 de Marzo de 2013) Acept (15 de Mayo de 2014)

\begin{abstract}
Resumen
Se plantea la adaptación y validación española de la versión breve de la Sexual Inhibition/Sexual ExcitationScales que evalúan la propensión hacia la excitación e inhibición sexuales. Fue contestada a través de Internet, junto a otras medidas de funcionamiento sexual, por 796 sujetos de la población general (18-84 años). El Análisis Factorial Exploratorio dio lugar a una estructura factorial similar a la original (Escala de Excitación Sexual, ESS; y dos Escalas de Inhibición Sexual, SIS1 y SIS2), que fue ratificada mediante Análisis Factorial Confirmatorio. Además, la escala resultó equivalente para hombres y mujeres al alcanzar un nivel de invarianza estricta. La consistencia interna es adecuada. La dimensión SES se relaciona positivamente al deseo sexual, mientras que SIS1 y SIS2 lo hacen de forma negativa. Las dos medidas de inhibición sexual se asocian a peor funcionamiento sexual. Los hombres muestran mayor puntuación en SES y las mujeres en SIS1.

Palabras clave: EIS/SES, excitación, inhibición, fiabilidad, validez.
\end{abstract}

\section{Abstract.}

This study proposes the adaptation and validation of the Spanish version of the Sexual Inhibition/Sexual Excitation Scales for the assessment of individual propensity for sexual excitation and sexual inhibition. The scale was located online and it was administered with other measures of sexual functioning. The questionnaires were answered by 796 individuals from the general population (18-84 years old). The Exploratory Factor Analysis yielded a factorial structure which was similar to the one proposed originally (Sexual Excitation Scale, SES; and two Sexual Inhibition Scales, SIS1 and SIS2), which was confirmed through Confirmatory Factor Analysis. The scale was equivalent between men and women, as it reached strict invariance. Internal consistency is adequate. The SES dimension is positively related to sexual desire, while SIS1 and SIS2 are negatively related to it. The two measures of sexual inhibition are associated with worse sexual functioning. Men show higher scores on SES and women on SIS1.

Keywords: SIS/SES, excitation, inhibition, reliability, validity. 


\section{Introducción}

El Modelo de Control Dual de la respuesta sexual sugiere que la excitación sexual es resultado del balance entre la excitación sexual (ES) y la inhibición sexual (IS), siendo ambos sistemas relativamente independientes (Bancroft, 1999; Bancroft y Janssen, 2000). Dos aspectos centrales de este modelo son, por un lado, el concepto de variabilidad individual, según el cual los individuos se distribuyen en un continuo de propensión hacia la inhibición o excitación de la respuesta sexual y, por otro, que la activación relativa de ambos sistemas da lugar a una excitación sexual que resultará adaptiva o por el contrario disfuncional. Así, Bancroft (1999) enumera las ocasiones en las que un determinado nivel de inhibición en el varón puede resultar adaptativo: 1) cuando una situación sexual es percibida como amenaza (e.g., riesgo de infecciones de transmisión sexual), 2) cuando la amenaza percibida no es sexual y la inhibición de otros patrones de respuesta que resultan distractores, como la sexual, es necesaria, 3) para asegurar que la búsqueda del placer sexual no resulte excesiva de modo que distraiga de otras funciones adaptativas importantes, 4) cuando las consecuencias de una continuada y "excesiva" conducta sexual incluye la reducción de fertilidad debido al exceso de eyaculación, y 5) cuando las presiones sociales o ambientales dan lugar a la supresión de la conducta reproductiva y a la reducción de la densidad de población. Es necesario considerar que determinados niveles de excitación/inhibición pueden no resultar adaptativos. Los individuos con elevados niveles de inhibición tienen mayor probabilidad de padecer una disfunción sexual, como problemas de erección (Bancroft y Janssen, 2000) o bajo deseo sexual (Bancroft, 1999; Prause, Janssen y Hetrick, 2008), y que elevados niveles de excitación sexual y bajos niveles de inhibición están asociados a conductas de mayor riesgo sexual al dificultar los procesos de toma de decisiones sexuales (Bancroft et al., 2004).

El Modelo de Control Dual distingue entre excitación sexual (ES) y dos tipos de inhibición sexual presentes tanto en hombres como en mujeres: una relacionada con el miedo a la ejecución sexual (IS1) y otra derivada del miedo a las consecuencias sexuales (IS2). En tanto que el primer tipo de inhibición sexual se ha considerado más como un rasgo, al considerarse un nivel "tónico" de inhibición sexual, el segundo depende del contexto y las circunstancias que envuelven a la actividad sexual (Bancroft y Janssen, 2000). Ambos son determinantes del funcionamiento sexual especialmente la IS1, que ha demostrado estar relacionada con la presencia de disfunciones sexuales. Asimismo, diversos estudios muestran que la ES está asociada con deseo sexual diádico y solitario, en tanto que la IS2 está negativamente asociada con ambos tipos de deseo en los dos sexos (Winters, Christoff y Gorzalka, 2008), lo que podría indicar que el deseo refleja procesos tempranos de la excitación (Everaerd, Laan, Both y Van der Velde, 2000). Por ello, la evaluación de ambos procesos (excitación e inhibición sexuales) resulta de importancia en el estudio del funcionamiento sexual, así como en la elección de enfoques terapéuticos idóneos dependiendo del tipo de inhibición sexual que el paciente presente (véase Janssen y Bancroft, 2007).

Con el objetivo de evaluar los procesos de excitación e inhibición sexuales se han desarrollado diversos instrumentos. En un primer momento se elabora el Sexual Inhibition/Sexual Excitation Scalespara varones (SIS/SES; Janssen et al., 2002a) con 45 ítems distribuidos en una escala de Excitación (SES), y dos escalas relacionadas con la Inhibición sexual (SIS1 y SIS2). Asimismo se generó un instrumento específico para mujeres, el Sexual Excitation/Sexual Inhibition Inventory for Women(SESII-W; Graham, Sanders y Milhausen, 2006). El SIS/SES (Janssen, Vorst, Finn y Bancroft, 2002a) fue validado en mujeres (Carpenter, Janssen, Graham, Vorst y Wicherts, 2008), aunque los autores recomiendan el uso del SIS/SES (Janssen et al., 2002a) y del SESII-W (Graham et al., 2006) cuando se evalúe a hombres y mujeres respectivamente. Debido a que en el SIS/SES (Janssen et al., 2002a) no todos los ítems resultaban igualmente relevantes para hombres y mujeres, se diseña una nueva versión equivalente para ambos sexos: el SexualInhibition/Sexual Excitation Scales-Short Form (SIS/SES-SF; Carpenter, Janssen, Graham, Vorst y Wicherts, 2011). Esta versión reducida resulta de una selección de los ítems del SIS/SES que mejor representan la estructura trifactorial en hombres y en mujeres (Carpenter et al., 2008; Janssen et al., 2002a).

El Sexual Inhibition/Sexual Excitation Scales-Short Form (SIS/SES-SF) [Escalas de Inhibición Sexual/Excitación Sexual-Forma Breve (SIS/SES-SF)] consta de 14 ítems distribuidos en tres factores: a) Escala de Excitación Sexual (SES) que evalúa la excitación sexual derivada de interacciones sociales (e.g., Cuando un/a desconocido/a sexualmente atractivo/a me toca accidentalmente, me excito con facilidad); b) Escala de Inhibición Sexual-1 (SIS1), que incluye ítems relacionados con la distracción/concentración en la ejecución sexual y problemas pasados relacionados con la excitación (e.g., No consigo excitarme a menos que me concentre exclusivamente en la estimulación sexual); y c) Escala de Inhibición Sexual-2 (SIS2), referidaal riesgo de ser descubierto o contraer algún tipo de infección de transmisión sexual o ITS (e.g., Si creo que otras personas pudieran verme mientras tengo relaciones sexuales, es poco 
probable que mantuviese la excitación). Su escala de respuesta es tipo Likert de 4 puntos ( 1 = Completamente de acuerdo a 4 = Completamente en desacuerdo). Sus puntuaciones correlacionan con la versión extensa del SIS/SES (Janssen et al., 2002) de 45 ítems en hombres: SES ( $r=.90)$, SIS1 $(r=.80)$ y SIS $2(r=.80)$. También se obtuvieron adecuados valores de fiabilidad test-retest: entre .61 del SES y SIS1 en mujeres y .75 del SES en varones.

El objetivo de este estudio instrumental fue adaptar y validar las Sexual Inhibition/Sexual Excitation Scales-Short Form (Escalas de Inhibición Sexual/Excitación SexualForma Breve, SIS/SES-SF) de Carpenter et al. (2011)en hombres y mujeres españoles. Se examinará su estructura factorial y equivalencia en hombres y mujeres, se calculará la consistencia interna y se buscarán evidencias acerca de la validez de sus medidas.Se plantean las siguientes hipótesis: 1) Se obtendrá una estructura trifactorial similar a la planteada originalmente por los autores, formada por un factor de excitación (SES) y dos de inhibición sexual (SIS1 y SIS2) (Carpenter et al., 2011; Janssen et al., 2002a), que resultará equivalente en hombres y mujeres; 2) se espera que los varones obtengan mayores puntuaciones en excitación sexual (SES) y las mujeres en inhibición sexual (SIS1 y SIS2) (Carpenter et al., 2008) y 3) la excitación sexual (SES) se asociará de forma positiva con deseo sexual (diádico y solitario), así como con un mejor funcionamiento sexual en cada una de sus áreas (deseo, excitación, orgasmo, erección -en los varones- y satisfacción sexual); mientras, la inhibición sexual (SIS1 y SIS2) se relacionará con un menor deseo sexual y peor funcionamiento sexual en cada área (Bancroft, Graham, Janssen y Sanders, 2009; Bancroft y Janssen, 2000).

\section{Método}

\section{Participantes}

Un total de 1.812 personas accedieron a la encuesta online, de los cuales 821 cumplimentaron toda la encuesta y cumplían con los requisitos de participación explicitados en el consentimiento informado ofrecido al inicio de la encuesta: ser mayor de 18 años, tener nacionalidad española y mantener una relación de pareja heterosexual de al menos seis meses con actividad sexual. Tras considerar la existencia de outliers, fueron eliminados 25 sujetos (14 hombres y 11 mujeres) por lo que la muestra final quedó constituida por 796 sujetos (45.6\% hombres y $54.4 \%$ mujeres) con un rango de edad entre 18 y 84 años $(M=32.67 ; D T=11.49)$. En cuanto al nivel de estudios, el $3 \%$ tenía estudios primarios, el $30.7 \%$ estudios secundarios y el $66.2 \%$ estudios superiores. El 48.6\% no profesaba ninguna religión, el $50.8 \%$ profesaba la religión cristiana y el $0.7 \%$ otra religión.

Para los análisis estadísticos, los participantes fueron divididos aleatoriamente en: 1 ) muestra 1 , formada edades entre sujetos ( $42.3 \%$ varones y $57.7 \%$ mujeres) con edades entre 18 y 84 años $(M=32.89 ; D T=11.54)$ para realizar el Análisis Factorial Exploratorio (AFE) y 2) muestra 2, integrada por 399 sujetos ( $48.9 \%$ varones y $51.1 \%$ mujeres) con un rango de edad de $18-82$ años $(M=32.46 ; D T=11.46)$ para realizar el Análisis Factorial Confirmatorio (AFC), no habiendo diferencias significativas entre ambas submuestras en cuanto a edad $(t(794)=.52 ; p=.60)$, sexo $\left(\mathrm{x}^{2}=3.44 ; p\right.$ $=.06)$, nivel de estudios $\left(\mathrm{x}^{2}=0.32 ; p=.57\right)$ ni religión $\left(\mathrm{x}^{2}\right.$ $=1.03 ; p=.31)$.

\section{Instrumentos}

Cuestionario sociodemográfico, que incluye preguntas acerca del sexo, edad, nivel de estudios y religión.

Versión española de las Sexual Excitation/Inhibition Scales-Short Form (Carpenter et al.,2011). Siguiendo las recomendaciones de los autores, las respuestas dadas a SIS/SES-SF fueron recodificadas, de modo que: $1=$ Completamente en desacuerdo, $2=$ En desacuerdo, $3=$ De acuerdo y 4 = Completamente de acuerdo.

Versión española del Inventario de Deseo Sexual (IDS; Spector, Carey y Steinberg, 1996) de Ortega, Zubeidat y Sierra (2006). Formada por 13 ítems que evalúan el interés por la actividad sexual diádica y solitaria. La adaptación española presenta valores alfa de Cronbach óptimos (.87 para deseo sexual diádico y.88 para deseo sexual solitario), así como adecuados indicadores de validez (Ortega et al., 2006). En el presente estudio el valor alfa de Cronbach para deseo sexual diádico fue .72 y .88 para deseo sexual solitario.

Versión española del Massachusetts General Hospital Sexual-Functioning Questionnaire (MGH-SFQ; Fava, Rankin, Alpert, Nierenberg y Worthington, 1998) de Sierra, VallejoMedina, Santos-Iglesias y Lameiras Fernández (2012), compuesta por cinco ítems que evalúan el funcionamiento sexual en el último mes en cinco áreas diferentes: interés o deseo sexual, excitación, orgasmo, erección (en varones) y satisfacción sexual. La suma de las puntuaciones de los cinco ítems (cuatro en el caso de las mujeres) ofrece una puntuación de funcionamiento sexual global. Sierra et al. (2012) informan de valores de consistencia interna de $.90 \mathrm{y}$ .93 en varones y mujeres, respectivamente, así como buenos 
indicadores de la validez. En la muestra de este estudio se obtuvo un coeficiente alfa de Cronbach igual a .80 tanto en hombres como en mujeres.

\section{Procedimiento}

En primer lugar se realizó la traducción hacia delante de los ítems de las Sexual Inhibition/Sexual Excitation ScalesShort Form del inglés al castellano de modo independiente por dos miembros del grupo de investigación con alto dominio del inglés, conocimiento de ambas culturas, e instruidos en psicometría y elaboración y construcción de cuestionarios. Posteriormente, las dos versiones traducidas fueron consensuadas para obtener una primera versión traducida. Esa versión inicial fue evaluada por un psicólogo bilingüe experto en sexualidad, quien con la versión original en inglés y la versión traducida al castellano realizó sugerencias en dos de los 14 ítems. Estas sugerencias fueron integradas en la nueva versión, que fue evaluada nuevamente a través de un juicio de expertos, a quienes se hizo entrega de la versión original en inglés y la versión traducida en castellano. Estos cinco expertos que examinaron la adaptación debían indicar si resultaba adecuada: a) la comprensión del ítem redactado en español y b) la equivalencia del contenido de los ítems traducidos al español y los ítems originales en inglés, así como ofrecer sugerencias o redacciones alternativas cuando lo considerasen. En los ítems para los que no se alcanzó un grado de acuerdo de al menos el $80 \%$ sobre la comprensión o equivalencia de contenido se realizaron cambios siguiendo las recomendaciones y sugerencias realizadas por los expertos, dando lugar a modificaciones en seis ítems. Tras este proceso de revisión, el cuestionario en castellano fue entregado a 20 hombres y 20 mujeres de la población general española para que considerasen si comprendían adecuadamente los ítems. Tras esta prueba piloto, uno de los ítems fue modificado obteniendo así la versión final del instrumento.

La batería de cuestionarios fue digitalizada en la plataforma LimeSurvey de la Universidad de Granada. El link de la encuesta fue http://encuestasexualidad.ugr.me. Se distribuyó a través de diversas redes sociales, correo electrónico, páginas web de la Universidad de Granada y diversos periódicos y revistas en Internet. La muestra fue recogida entre los meses de mayo y agosto de 2012. Una vez el participante accedía a la encuesta online, se le presentaba un consentimiento informado que incluía el objetivo general del estudio y los requisitos necesarios de participación, así como la Ley de Protección de Datos de Carácter Personal en España 15/1999, garantizándose el anonimato y confidencialidad de las respuestas. La participación fue voluntaria, sin recibir ningún tipo de compensación.Todos los cuestionarios constaban de instrucciones que facilitasen su cumplimentación. El tiempo estimado para la realización de la encuesta fue de aproximadamente 30 minutos, sin embargo, el participante tenía la opción de salir y reiniciar la encuesta posteriormente.

\section{Resultados}

\section{Análisis factorial exploratorio (AFE)}

Para examinar la estructura factorial del instrumento se realizó un AFE en la primera submuestra $(n=397)$. Para ello, se comprobó la adecuación de los datos para realizar el proceso de factorialización a través de la prueba KMO $=0.75 \mathrm{y}$ esfericidad de Barlett $\left(\mathrm{x}^{2}=1158.55 ; p=.000\right)$. Se realizó el AFE a través de componentes principales, en el que se prefijó una estructura de tres factores, similar a la estructura de la versión original: un factor de excitación sexual (SES) y dos factores de inhibición sexual (SIS1 y SIS2). Se empleó la rotación Varimax. La estructura factorial resultante explicó un $48.50 \%$ de la varianza total. Como se observa en la Tabla 1, todas las saturaciones fueron significativas y oscilaron entre .53 y .79. En todos los factores los eigenvalues superaron el valor $1 \mathrm{y}$ las comunalidades de los ítems fueron superiores a .33 , valor más bajo que se obtuvo en los ítems 3 y 9. Las correlaciones ítem-total corregidas en cada factor oscilaron entre .28 del ítem 6 (SIS2) y .64 del ítem 14 (SES). La estructura factorial quedó configurada del siguiente modo: 1) Escala de Excitación Sexual (SES); explica el $21.50 \%$ de la varianza, estando formado por los ítems $1,3,8,10,11$ y 14 , y hace referencia a la excitación sexual derivada de las interacciones sociales. 2) Escala de Inhibición Sexual-1 (SIS1), que explica el $18.02 \%$ de la varianza, agrupa los ítems 4, 9, 12 y 13, y alude a la distracción/concentración en la ejecución sexual y problemas en el pasado relacionados con la excitación. 3) Escala de Inhibición Sexual-2 (SIS2), explica el 8.95\% de la varianza y está compuesto por los ítems 2, 5, 6 y 7; se refiere a temas relacionados con el riesgo de ser descubierto durante la actividad sexual o de contraer una infección de transmisión sexual.

Se obtuvieron correlaciones significativas entre los factores, excepto entre SES y SIS1, demostrándose independencia entre ambos; la correlación entre SES y SIS2 fue baja ( $r=$ -.12; $p<.01)$. La correlación entre SIS1 y SIS2 fue de .43 
Tabla 1.Matriz de cargas factoriales

Factores e ítems

Factor 1: Escala de Excitación Sexual (SES)

14. Cuando una persona atractiva coquetea conmigo, me excito fácilmente

8. Cuando pienso en una persona muy atractiva, me excito fácilmente

1. Cuando un/a desconocido/a sexualmente atractivo/a me toca accidentalmente, me excito con facilidad

11. Cuando veo a otros teniendo contactos sexuales, me dan ganas de tener sexo

3. Cuando hablo por teléfono con alguien que tiene una voz sexy, me excito sexualmente

10. Cuando comienzo a tener fantasías sexuales, me excito rápidamente

Factor 2: Escala de Inhibición Sexual 1 (SIS1)

13. Si me distraigo por el sonido de música, televisión o conversación es poco probable que mantenga la excitación $\quad .74$

12. Cuando algún pensamiento me distrae, pierdo fácilmente mi erección / excitación 72

4. No consigo excitarme a menos que me concentre exclusivamente en la estimulación sexual

9. Una vez que consigo una erección (o estoy excitada) quiero comenzar el coito inmediatamente, antes de que pudiera perder mi erección (o excitación)

Factor 3: Escala de Inhibición Sexual 2 (SIS2)

6. Si me doy cuenta de que hay riesgo de contraer una infección de transmisión sexual, es poco probable que mantenga la excitación sexual

5. Si me estoy masturbando a solas y me doy cuenta de que alguien puede entrar en la habitación en cualquier momento, perderé mi erección/excitación sexual

2. Si tengo sexo en un lugar al aire libre, aislado, y creo que hay alguien cerca, es difícil que me excite mucho

7. Si creo que otras personas pudieran verme mientras tengo relaciones sexuales, es poco probable que mantuviese la excitación

Tabla 2. Estadísticos descriptivos de la solución factorial de 3 factores $(n=397)$

\begin{tabular}{|c|c|c|c|c|c|c|c|c|c|}
\hline Factor & Ítem & $\begin{array}{c}\% \\
\text { varianza }\end{array}$ & $M$ & $D T$ & Rango & Asimetría & Curtosis & $\begin{array}{l}\text { Item-total } \\
\text { corregida }\end{array}$ & $\alpha^{*}$ \\
\hline \multirow[t]{7}{*}{ SES } & & 21.43 & 2.74 & 0.48 & $1.33-4$ & -.03 & -.08 & & .72 \\
\hline & 1 & & 2.37 & 0.89 & & .07 & -.76 & .50 & .67 \\
\hline & 3 & & 2.24 & 0.79 & & .12 & -.51 & .37 & .71 \\
\hline & 8 & & 2.69 & 0.71 & & .00 & -.35 & .58 & .65 \\
\hline & 10 & & 3.20 & 0.61 & & -.15 & -.52 & .32 & .72 \\
\hline & 11 & & 3.16 & 0.68 & & -.61 & .67 & .36 & .71 \\
\hline & 14 & & 2.75 & 0.72 & & -.04 & -.37 & .64 & .63 \\
\hline \multirow[t]{5}{*}{ SIS1 } & & 18.12 & 2.19 & 0.54 & $1-4$ & .15 & .26 & & .66 \\
\hline & 4 & & 1.97 & 0.77 & & .46 & -.17 & .38 & .64 \\
\hline & 9 & & 2.13 & 0.80 & & .40 & -.20 & .36 & .65 \\
\hline & 12 & & 2.40 & 0.71 & & -.07 & -.31 & .54 & .54 \\
\hline & 13 & & 2.25 & 0.78 & & .26 & -.25 & .51 & .55 \\
\hline \multirow[t]{5}{*}{ SIS2 } & & 8.72 & 2.84 & 0.59 & $1-4$ & -.20 & -.14 & & .65 \\
\hline & 2 & & 2.60 & 0.87 & & -.11 & -.66 & .48 & .55 \\
\hline & 5 & & 2.72 & 0.88 & & -.24 & -.63 & .44 & .58 \\
\hline & 6 & & 3.46 & 0.72 & & -1.21 & .85 & .28 & .67 \\
\hline & 7 & & 2.60 & 0.88 & & .01 & -.76 & .53 & .51 \\
\hline
\end{tabular}

* Alfa de Cronbach si el ítem es eliminado 
$(p<.001)$, lo que demuestra cierto solapamiento de ambas subescalas de inhibición.

En la Tabla 2 se presentan los estadísticos descriptivos. Los valores de asimetría y curtosis se consideraron normales ya que adoptaron valores que oscilaron entre $-1 \mathrm{y}+1 \mathrm{y}$ entre -1 y +2 , respectivamente (Huck, 2000), a excepción del ítem 6 ("Si me doy cuenta de que hay riesgo de contraer una infección de transmisión sexual, es poco probable que mantenga la excitación sexual') que presentó un valor de asimetría igual a 1.21.La media obtenida en este ítem fue 3.46, indicando que la mayoría de puntuaciones se sitúan entre las opciones de respuesta 3-4 (De acuerdo, Completamente de acuerdo). El valor alfa de Cronbach al eliminar el ítem 6 del factor SIS2, pasaría de .64 a .67, no considerándose este aumento considerable.Estos resultados podrían deberse a que el contenido de este ítem en comparación con el resto de ítems del factor es diferente. En tanto que los ítems 2, 5 y 7 hacen referencia a la inhibición sexual producida por la posibilidad de ser pillado durante la actividad sexual, el ítem 6 se refiere al riesgo de contraer una enfermedad de transmisión sexual. La correlación ítem-total corregida fue superior a .30 por lo que no se planteó la eliminación del ítem 6 de modo previo a la confirmación del modelo.

\section{Análisis factorial confirmatorio (AFC)}

Se llevó a cabo un AFC en la segunda submuestra ( $n=$ 399) a través de AMOS 7.0. Debido al incumplimiento de normalidad de los datos, mediante Kolmogorov-Smirnov $(\mathrm{p}<.001)$ se utilizó el método de Generalized Least Squares (GLS). Se puso a prueba el modelo de tres factores propuesto originalmente por los autores y que fue obtenido previamente a través de AFE en este mismo estudio. Se emplearon los siguientes índices de bondad de ajuste: el ratio $\chi 2 / g 1$, GFI, AGFI y RMSEA. Para el ratio $\chi 2 / g 1$, valores inferiores a 3 indican un buen ajuste (Chau, 1997). Valores mayores o iguales a .85 en GFI y AGFI son igualmente indicadores de ajuste adecuado (Jöreskog y Sörbom, 1993) y para RMSEA, un valor inferior a .05 indicaría un modelo con buen ajuste (Schumacker y Lomax, 2004). Para el establecimiento del modelo, se fijó a 1 uno de los indicadores en cada factor. En la Tabla 3 se muestra que todos los índices de ajuste obtenidos en el modelo fueron óptimos. En la Figura 1 aparece el path diagram del modelo con los respectivos pesos estandarizados que oscilaron entre .33 (ítem 6) y .80 (ítem 14), siendo las correlaciones entre factores de -.016 entre SES y SIS1, -.22 entre SES y SIS2, y de .61 entre SIS1 y SIS2, con una media de correlación entre factores de .28. Correlaciones múltiples cuadradas oscilaron entre .10 del ítem 6 hasta .64 del ítem 14, siendo la media de .34, indicando que el $34 \%$ de la varianza es explicada por las variables latentes.

Para conocer si la estructura factorial resulta equivalente en hombres y mujeres, las puntuaciones se pusieron a prueba a través de la invarianza de sus medidas, siguiéndose las recomendaciones de Byrne (2008). Se usó el método Generalized Least Squares. Se consideró indicio de invarianza que el $C F I$ no disminuyese más de .01 con respecto al modelo anterior (Cheung y Rensvold, 2002), así como el incremento de $\mathrm{x} 2 / \mathrm{gl}$ entre los modelos propuestos con respecto al anterior. Se alcanzó invarianza estricta, por lo que se asegura que el instrumento pueda utilizarse en ambos sexos (véase la Tabla 4).

\section{Diferencias entre hombres y mujeres en la SIS/SES- SF}

Como se observa en la Tabla 5, se llevó a cabo MANCOVA, controlando la edad, ya que la muestra utilizada cubre un amplio intervalo de edad (desde 18 hasta 84 años) y estudios previos indican que la excitación sexual puede verse influida por este factor (Bancroft y Janssen, 2000; Graham et al., 2006). Los hombres obtuvieron puntuaciones más altas en excitación sexual (SES), en tanto que las mujeres puntuaron significativamente más alto en inhibición sexual, en concreto en SIS1.

Tabla 3: Índices de bondad de ajuste obtenidos a través de AFC

\begin{tabular}{lcccccc}
\hline & $\mathrm{x}^{2}$ & $g l$ & $\mathrm{x}^{2 /} \mathrm{gl}$ & RMSEA & GFI & AGFI \\
\hline Modelo de tres factores & 152.94 & 74 & 2.06 & 0.05 & .94 & .92 \\
\hline
\end{tabular}




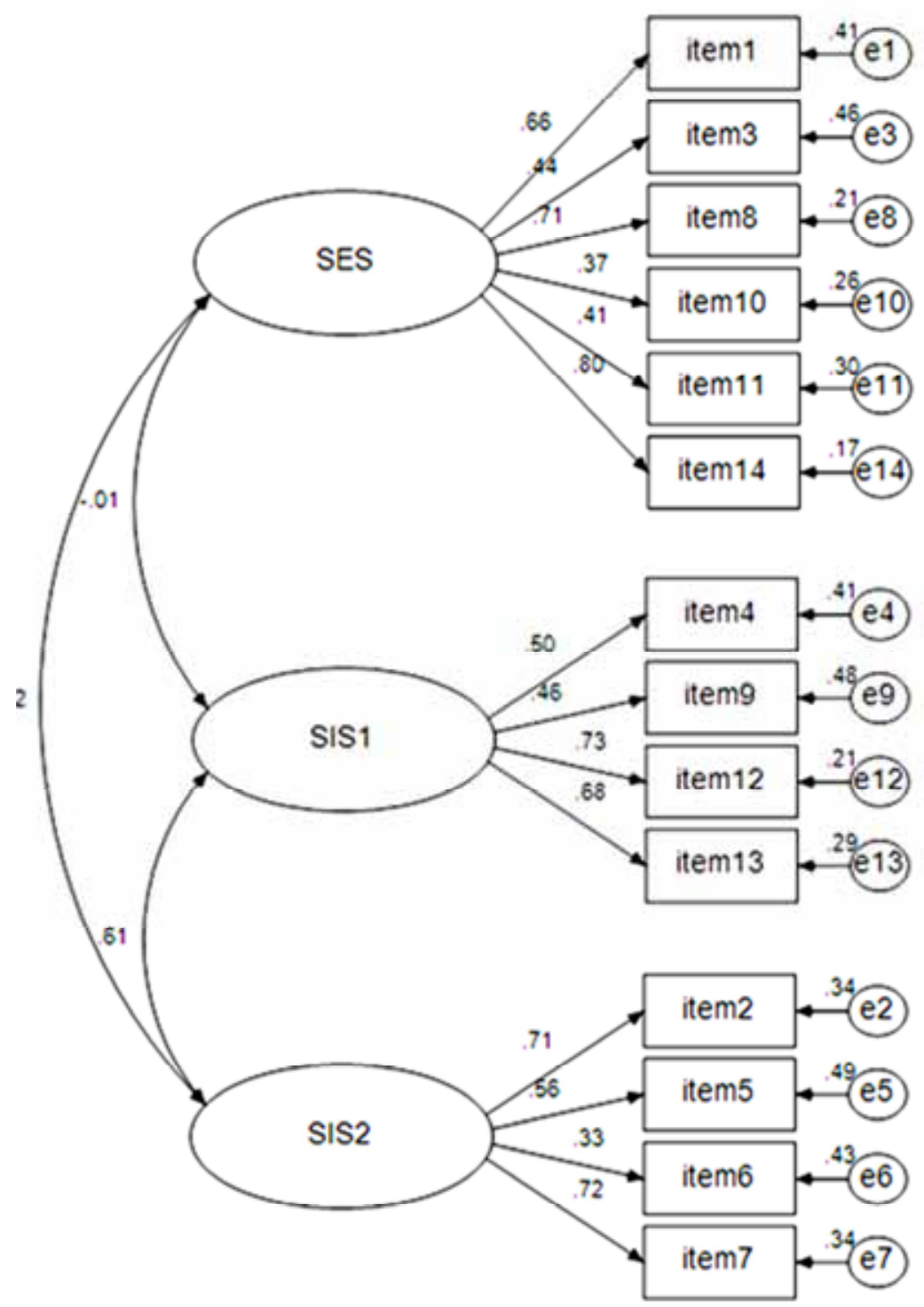

Figura 1. Path diagram del modelo de tres factores

Tabla 4: Índices de bondad de ajuste de los modelos de invarianza

\begin{tabular}{lllll}
\hline & $\mathrm{x}^{2}$ & $\mathrm{gl}$ & RMSEA & CFI \\
\hline $\begin{array}{l}\text { Invarianza configural } \\
\text { (sin restricciones) }\end{array}$ & 222.39 & 151 & 0.035 & 0.754 \\
Igual saturación en los factores & 236.07 & 162 & 0.034 & 0.744 \\
Igual covarianza estructural & 240.45 & 165 & 0.034 & 0.740 \\
Igual varianza de errores & 256.52 & 179 & 0.033 & 0.733 \\
\hline
\end{tabular}


Tabla 5: Diferencias entre hombres y mujeres en cada factor, controlando edad

\begin{tabular}{lcccc}
\hline Factor & $M$ & $D T$ & $t$ & d de Cohen \\
\hline 1. SES & & & $5.59^{* *}$ & 0.23 \\
Varones & 2.78 & 0.47 & & \\
Mujeres & 2.67 & 0.48 & & 0.28 \\
2. SIS1 & & & $27.76^{* * *}$ & \\
Hombres & 2.10 & 0.56 & & 0.08 \\
Mujeres & 2.25 & 0.51 & 3.99 & \\
3. SIS2 & & & \\
Hombres & 2.80 & 0.57 & & \\
Mujeres & 2.85 & 0.58 & & \\
\hline
\end{tabular}

$* * * p<.001 ; * * p<.01 ; * p<.05$

Varones

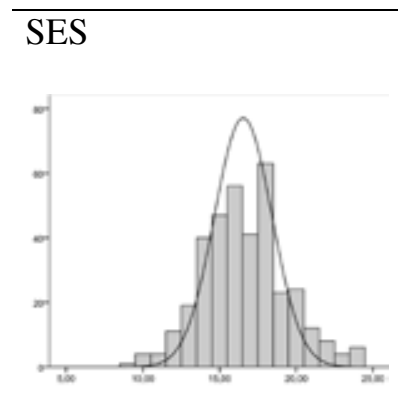

$M(D T)=16.65(2.75)$

Rango $=9-24$

Alfa $=.72$

Mujeres

\section{SES}

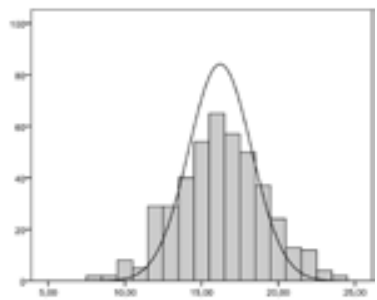

$M(D T)=16.24(2.89)$

Rango $=8-24$

Alfa $=.71$

\section{SIS 1}

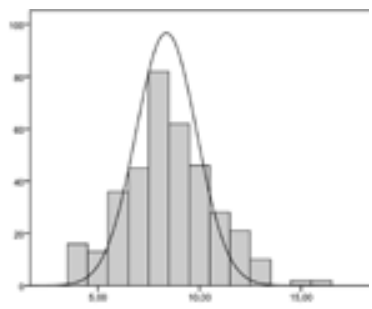

$M(D T)=8.50(2.21)$

Rango $=4-16$

Alfa $=.69$
SIS2

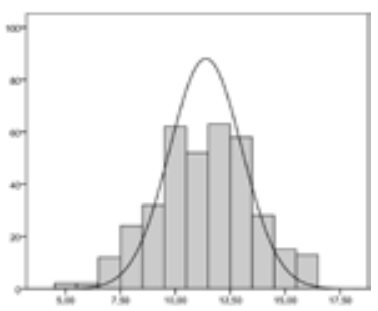

$M(D T)=11.33(2.25)$

Rango $=5-16$

Alfa $=.60$
SIS1

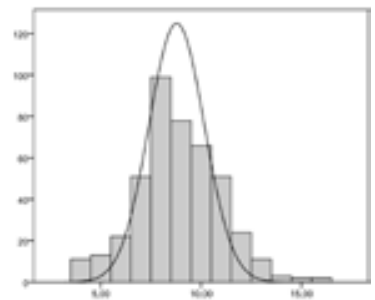

$M(D T)=8.89(2.08)$

Rango $=4-16$

Alfa $=.64$
SIS2

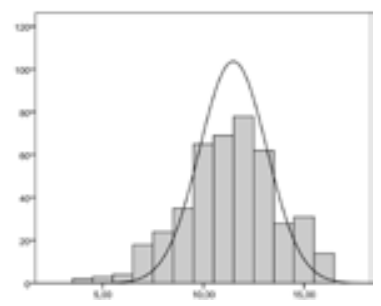

$M(D T)=11.34(2.35)$

Rango = 4-16

Alfa $=.62$

Figura 2: Distribución de las puntuaciones en SIS/SES-SF en hombres $(n=363)$ y mujeres $(n=433)$ 


\section{Fiabilidad y validez}

Con la totalidad de la muestra $(\mathrm{N}=796)$ se obtuvieron los valores alfa de Cronbach (véase la Figura 2) y evidencias de validez, controlándose el efecto de la edad, con deseo sexual diádico, deseo sexual solitario y áreas del funcionamiento sexual en el último mes. Los análisis se realizaron independientemente en hombres y mujeres (Carpenter et al., 2011). Los resultados se presentan en la Tabla 6.

\section{Discusión}

Con el objetivo de adaptar y validar las SIS/SES-SF (Carpenter et al., 2011) en población española se llevó a cabo el proceso de adaptación del inglés al castellano de los ítems y posteriormente la estructura factorial del instrumento se puso a prueba a través de AFE en una muestra de 397 sujetos, ratificándose sus tres factores en una muestra de 399 sujetos a través de AFC. De igual manera que ocurría con la versión original (Carpenter et al., 2011) se obtiene un factor de excitación sexual derivada de las interacciones sociales (SES) y dos factores de inhibición sexual, siendo SIS1 relativo a la inhibición sexual por distracción/concentración en las relaciones sexuales y SIS2 referente al riesgo de ser pillado por otras personas durante la actividad sexual o de contraer una infección de transmisión sexual. Los tres factores explican conjuntamente el $48.47 \%$ de la varianza. Asimismo, se ha obtenido una relación negativa entre SES y SIS2 y una relación positiva entre SIS1 y SIS2, demostrándose cierto solapamiento en el constructo que evalúan. Sin embargo, no se encontró relación entre SES y SIS1, por lo que ambos factores resultan independientes. Los resultados difieren parcialmente de los de estudios previos en los que se muestra independencia entre SES y SIS2, aunque sí se mantiene la relación entre los dos factores de inhibición sexual (Janssen et al., 2002a).

La escala, formada por 14 ítems, resulta equivalente en hombres y mujeres al alcanzar un nivel de invarianza estricta, por lo que su aplicación en ambos sexos resulta adecuada. Asimismo, las respuestas ofrecidas en cada factor en ambos sexos siguen un patrón de distribución normal. Las comparaciones realizadas en cada uno de los factores entre hombres y mujeres muestran resultados similares a los de Carpenter et al. (2008), ya que los primeros presentan puntuaciones superiores en SES, en tanto que las mujeres informan de mayores puntuaciones en SIS1. No existen diferencias en SIS2. Esta mayor propensión de excitación sexual en el varón podría explicar la existencia de mayores niveles de deseo sexual, como ha sido ampliamente demostrado (Baumeister, Catanese y Vohs, 2001; Giargiari, Mahaffey, Craighead y Hutchison, 2005; Impett y Peplau, 2003; Petersen y Hyde, 2011), que a su vez es expresado a través de diversas manifestaciones (Levine, 2010; SantosIglesias, Calvillo y Sierra, 2013) como es la frecuencia de fantasías sexuales (Hicks y Leitenberg, 2001; Moyano y Sierra, 2013), mayor inicio de conductas sexuales con la pareja (Santos-Iglesias y Sierra, 2010, 2012) o una elevada frecuencia de masturbación (Das, 2007; Sierra, Perla y Gutiérrez-Quintanilla, 2010; Van Anders, 2012). Sin embargo, las mujeres están más predispuestas hacia la inhibición sexual relacionada con la distracción/concentración durante

Tabla 6: Correlaciones parciales, controlando edad, de SES, SIS1 y SIS2 con otras medidas en hombres y mujeres $(\mathrm{N}=796)$

\begin{tabular}{lllllll}
\hline \multicolumn{1}{l}{ SES } & \multicolumn{3}{c}{ SIS1 } & \multicolumn{3}{c}{ SIS2 } \\
& Hombres & Mujeres & Hombres & Mujeres & Hombres & Mujeres \\
\hline Deseo diádico & $.20^{* * *}$ & $.29 * * *$ & $-.18^{* * *}$ & $-.22^{* * *}$ & $-.13^{*}$ & $-.18^{* * *}$ \\
Deseo solitario & $.33^{* * *}$ & $.32 * * *$ & .01 & $-.12^{*}$ & -.09 & $-.22^{* * *}$ \\
Func. global & -.03 & -.01 & $-.23^{* * *}$ & $-.22^{* * *}$ & $-.12^{*}$ & $-.14^{* *}$ \\
Deseo & .06 & -.00 & $-.14^{* *}$ & $-.16^{* *}$ & $-.13^{*}$ & $-.10^{*}$ \\
Excitación & -.01 & -.01 & $-.23^{* * *}$ & $-.24^{* * *}$ & -.08 & $-.12^{*}$ \\
Orgasmo & -.10 & .02 & $-.22^{* * *}$ & $-.19^{* * *}$ & $-.12^{*}$ & $-.12^{* *}$ \\
Erección & -.07 & - & $-.21 * * *$ & - & -.05 & - \\
Satisfacción & -.03 & -.04 & -.10 & $-.11^{*}$ & -.08 & $-.10^{*}$ \\
\hline
\end{tabular}

$* * * \mathrm{p}<.001 ; * * \mathrm{p}<.01 ; * \mathrm{p}<.05$ 
la actividad sexual. Investigaciones previas evidencian que las mujeres tienen más facilidad para inhibir de manera abrupta su excitación sexual (Graham et al., 2006; Simms y Meana, 2010), especialmente en determinadas situaciones que resultan amenazantes y que conciernen a su reputación (Tiefer, 2001), imagen corporal (Taylor, Rosen y Leiblum, 1994) o miedos relacionados con el embarazo (Sprecher y Regan, 1996), aunque en el presente estudio los valores alcanzados en SIS2 concerniente a las amenazas externas son similares en ambos sexos. Esta similitud podría deberse a la asimetría que presenta en el factor el ítem 6, indicando elevadas puntuaciones. Los indicadores de fiabilidad son adecuados en ambos sexos, alcanzándose valores alfa de Cronbach que oscilan entre .60 de SIS2 y .72 de SES, ambos en varones.

En cuanto a las evidencias de validez, se realizaron correlaciones parciales entre los tres factores de las SES/ SES y los diferentes componentes de la respuesta sexual. La edad fue controlada, dado su efecto sobre los mecanismos centrales excitatorios e inhibitorios (Janssen et al., 2002a), así como en la aparición de diversas dificultades sexuales como disfunción eréctil en los varones o sequedad vaginal en las mujeres (Araujo, Durante, Feldman, Goldstein y McKinlay, 1998; Bartlik y Goldberg, 2000). El factor SES se asoció al deseo sexual diádico y solitario en ambos sexos, de modo consistente con investigaciones previas en las que la excitación sexual y el deseo han mostrado relación (Bancroft y Vukadinovic, 2004; Janssen y Bancroft, 2007; Santos-Iglesias y Sierra, 2013; Winters et al., 2008). Este hecho está en la línea de las recientes propuestas sobre la dificultad por diferenciar el deseo o interés sexual de la excitación sexual (véase Brotto, 2009; Moyano y Sierra, en prensa), así como lo apuntado por diversos estudios que informan que el deseo y la excitación sexual cocvarían (Bozman y Beck, 1991; Rosen et al., 2000; Slob, Bax, Hop, Rowland y van der Werff ten Bosch, 1996), llegando incluso a considerarse el deseo sexual como la toma de conciencia de la excitación (Everaerd y Both, 2001). Como cabría esperar, tanto SIS1, cuyos ítems aluden al miedo a la ejecución sexual, como SIS2, relacionado con las amenazas externas, aparecen asociados a un menor deseo sexual diádico en ambos sexos. Asimismo, la propensión hacia la inhibición sexual interfiere en el deseo sexual solitario en la mujer, pero no en el varón. Los resultados dan cuenta por un lado de la distinción existente entre deseo sexual diádico y solitario pues ambos aspectos no resultan un componente unitario subyacente del "deseo" (Van Anders, 2012). Por otro lado, se considera la posibilidad de que determinadas conductas sexuales que se presentan con relativa constancia o estabilidad, como es el caso de la masturbación en los varones (Peplau, 2003), puedan no verse afectadas por la inhibición sexual, además de que el contenido de los factores de inhibición hace referencia a aspectos que surgen durante la actividad sexual en pareja. Sin embargo, en la mujer, la propensión hacia la inhibición sexual interfiere en la actividad sexual que ocurre en ausencia de otra persona, lo que podría quizás explicar que el deseo sexual en solitario de las mujeres sea generalmente más bajo que el de los varones, llegando incluso a ser similar al que informan personas que se identifican como asexuales (Prause y Graham, 2007), o bien que la inhibición sexual podría adquirir un mayor impacto sobre conductas que se realizan con poca frecuencia o que son más variables. En general, los resultados indicarían que la inhibición sexual de la mujer actúa de manera más global u holística, lo que podría reflejarse en la mayor prevalencia de deseo sexual hipoactivo en las mujeres, como informan encuestas realizadas en Estados Unidos (Laumann, Gagnon, Michael y Michaels, 1994) o España (Sierra et al., 2012). Sin embargo, la inhibición sexual en los varones parece interferir especialmente en interacciones sexuales con otras personas (Sapien y Córdoba, 2011), no viéndose afectada su sexualidad en solitario. Este indicador permite además realizar un mejor pronóstico sobre la posible eficacia terapéutica, ya que como observan Bancroft et al. (2005) en pacientes con disfunción eréctil, aquellos que responden mejor durante la masturbación tienen menos probabilidad de presentar un deterioro general de excitabilidad, siendo su disfunción de tipo "situacional".

Bancroft (1999) y Bancroft y Janssen (2000) teorizan que la propensión hacia la excitación e inhibición podrían ser determinantes importantes del funcionamiento sexual. En el presente estudio, la dimensión SES no se relaciona con ningún aspecto del funcionamiento sexual. Ahora bien, los resultados hallados indican que la inhibición sexual se asocia a un peor funcionamiento sexual, especialmente la que tiene que ver con la concentración/distracción durante las relaciones sexuales (SIS1). Este tipo de inhibición dificultaría el deseo, la excitación y el orgasmo en ambos sexos, así como la erección en varones. La satisfacción sexual es afectada sólo en la mujer. Los estudios basados en el Modelo de Control Dual han arrojado resultados inconsistentes sobre la influencia de la inhibición sexual en el funcionamiento (Bancroft et al., 2005), demostrando solo mayores niveles en SIS1 en varones con disfunción eréctil (Bancroft et al., 2009), no probándose dicha relación en mujeres con disfunciones sexuales. Empero, los resultados del presente estudio parecen dar apoyo a la relación entre inhibición y disfuncionalidad en ambos sexos. Estudios 
anteriores demuestran que la focalización en el rendimiento sexual, que caracteriza a SIS1, da lugar a la ansiedad de ejecución (Dove y Wiederman, 2000; McCabe, 2005) que suele desencadenar la aparición de ciertas disfunciones sexuales. En concreto, las mujeres que informan distraerse durante las interacciones sexuales (debido a preocupaciones relacionadas con la ejecución o la imagen corporal), suelen indicar tener orgasmos menos consistentes y menor satisfacción sexual (Dove y Wiederman, 2000), así como afectación de su excitación subjetiva (Salemink y Van Lankveld, 2006). Asimismo, el exceso de auto focalización impacta negativamente sobre la excitación sexual genital y subjetiva de la mujer (Van Lankveld y Bergh, 2008). Por otro lado, la inhibición sexual debido al riesgo de ser pillado durante un acto sexual o de contraer una infección de transmisión sexual se asocia a peor funcionamiento sexual en hombres y mujeres, a excepción de la dimensión excitación y erección, ambas en varones. Parece evidenciarse que los aspectos contextuales o situacionales adquieren mayor importancia en la sexualidad femenina (Bello-Villanueva, Palacio y Rodríguez-Díaz, 2013; Graziottin, Serafini y Palacios, 2009; Ramiro, Teva, Bermúdez y Buela-Casal, 2013; Rodríguez-Franco et al., 2012; Sarasúa, Zubizarreta, de Corral y Echeburúa, 2012; Wylie y Mimoun, 2009), ya que mayores niveles en SIS2 tienen un efecto en todas las fases de la respuesta sexual así como en su satisfacción en comparación con los hombres. Aunque se ha demostrado que ambos factores de inhibición sexual forman parte de un mismo constructo, SIS1 parece resultar más importante en la presencia de disfuncionalidad, por lo que es necesario examinar los efectos de cada uno de ellos de modo distinguible. Mientras SIS1 es considerado un "tono inhibitorio" (Bancroft y Janssen, 2000), la dimensión SIS2, relacionada con la posible aparición de amenazas externas, ha estado más vinculada con conductas sexuales de riesgo, que con problemas o dificultades sexuales (Bancroft et al., 2009). Sin embargo, la validez de SIS1 con medidas psicofisiológicas aún no ha sido demostrada (Janssen, Vorst, Finn y Bancroft, 2002b).

En conclusión, la versión breve del SIS/SES resulta una medida con adecuadas garantías psicométricas y de gran utilidad, especialmente al demostrar su equivalencia en hombres y mujeres, y permitir con ello realizar comparaciones entre ambos sexos. Asimismo, la relación demostrada entre los factores de inhibición con diversos aspectos del funcionamiento sexual contribuyen a dar apoyo a los planteamientos teóricos del Modelo de Control Dual de Bancroft (1999) y Bancroft y Janssen (2000), permitiendo la posibilidad de explorar su aplicación en individuos con disfunciones sexuales.

Ciertas limitaciones deben ser comentadas. En lo referente a la posible generalización de los datos, aunque el rango de edad fue amplio, la edad media de los participantes se corresponde a una población joven y con estudios universitarios, siendo difícil la generalización de los resultados. Para la evaluación del funcionamiento sexual, se ha utilizado un instrumento que evalúa cada una de sus áreas con un único ítem. Diversos autores indican que para una adecuada valoración de la disfuncionalidad y su severidad se requiere de la aplicación de instrumentos multi-ítem (Hayes, Dennerstein, Bennett y Fairley, 2008). Futuros estudios podrían además enriquecerse de la inclusión de medidas diferentes para la evaluación del funcionamiento sexual en varones y mujeres (Rizvi, Yeung y Kennedy, 2011). Por último, futuros estudios deberán poner a prueba la validez del Modelo de Control Dual con medidas psicofisiológicas, así como su aplicación en muestras clínicas.

\section{Referencias}

Araujo A. B., Durante, R., Feldman, H. A., Goldstein, I., y McKinlay, J. B. (1998). The relationship between depressive symptoms and male erectile dysfunction: cross-sectional results from the Massachusetts Male Aging Study. Psychosomatic Medicine, 60, 458-465.

Bancroft, J. (1999). Central inhibition of sexual response in the male: A theoretical perspective. Neuroscience and Biobehavioral Reviews, 23, 763-778. doi:10.1016/S0149-7634(99)00019-6.

Bancroft, J., Graham, C. A., Janssen, E., y Sanders, S. A. (2009). The Dual Control Model: Current status and future directions. Journal of Sex Research, 46, 121-142. doi:10.1080/00224490902747222.

Bancroft, J., Hederick, D., Barnes, T., Hallam-Jones, R., Wylie, K., ... Janssen, E. (2005). The relevance of the dual control model to male sexual dysfunction: the Kinsey Institute/BASRT collaborative project. Sexual and Relationship Therapy, 20, 13-30. doi:10.1080/146819905 12331298275.

Bancroft, J., y Janssen, E. (2000). The dual control model of male sexual response: A theoretical approach to centrally mediated erectile dysfunction. Neuroscience and Biobehavioral Reviews, 24, 571-579. doi:10.1016/S0149-7634(00)00024-5.

Bancroft, J., Janssen, E., Carnes, L., Strong, D. A., Goodrich, D., y Long, J. S. (2004). Sexual activity and risk-taking in young heterosexual men: The relevance of sexual arousability, mood, and sensation seeking. The Journal of Sex Research, 41, 181-192.

Bancroft, J., y Vukadinovic, Z. (2004). Sexual addiction, sexual compulsivity, sexual impulsivity, or what? Toward a theoretical model.The Journal of Sex Research, 41, 225-234.

Bartlik, B., y Goldberg, J. (2000). Female sexual arousal disorder. En S. R. Leiblum y R. C. Rosen (Eds.), Principles and practice of sex therapy (pp. 85-117). New York: Guilford Press.

Baumeister, R., Catanese, K., y Vohs, K. (2001). Is there a gender difference in strength of sex drive? Theoretical views, conceptual distinctions, and a review of relevant evidence. Personality and Social Psychology Review, 5, 242-273.

Bello-Villanueva, A. M., Palacio, J., y Rodríguez-Díaz, M. (2013). Medición de la intención en la actividad sexual en adolescentes: una 
aproximación de acuerdo al género del Caribe colombiano. Terapia Psicológica, 31, 343-353.

Bozman, A. W., y Beck, J. G. (1991). Covariation of sexual desire and sexual arousal: The effects of anger and anxiety. Archives of Sexual Behavior, 20, 47-60.

Brotto, L. A. (2009). The DSM diagnostic criteria for hypoactive sexual desire disorder in women. Archives of Sexual Behavior, 39, 221-239. doi:10.1007/s10508-009-9543-1.

Byrne, B. (2008). Testing for multigroup equivalence of a measuring instrument: A walk through the process. Psicothema, 20, 872-882.

Carpenter, D. L., Janssen, E., Graham, C. A., Vorst, H., y Wicherts, J. (2008). Women's scores on the Sexual Inhibition/Sexual Excitation Scales (SIS/SES): Gender similarities and differences. The Journal of Sex Research, 45, 36-48. doi:10.1080/00224490701808076.

Carpenter, D. L., Janssen, E., Graham, C. A., Vorst, H., y Wicherts, J. (2011). The Sexual Inhibition/Sexual Excitation Scales-Short Form (SIS/SES-SF). En T. D. Fisher, C. M. Davis, W. L. Yarber y S. L. Davis (Eds.), Handbook of Sexuality-Related Measures (pp. 236-239). London, UK: Routledge.

Chau, Y. K. (1997). Re-examining a model for evaluating information center success using a structural equation modelling approach. Decision Sciences, 28, 309-334.

Cheung, G. W., y Rensvold, R. B. (2002). Evaluating goodness-of-fit indexes for testing measurement invariance. Structural Equation Modeling, 9, 235-255.

Das, A. (2007). Masturbation in the United States. Journal of Sex and Marital Therapy, 33, 301-317. doi:10.1080./00926230701385514.

Dove, N. L., y Wiederman, M. W. (2000). Cognitive distraction and women's sexual functioning. Journal of Sex and Marital Therapy, 26, 67-78.

Everaerd, W., y Both, S. (2001). Ideal female sexual function. Journal of Sex and Marital Therapy, 27, 137-139.

Everaerd, W., Laan, E., Both, S., y Van der Velde, J. (2000). Female sexuality. En L. Szuchman y F. Muscarella (Eds.), Psychological perspectives on human sexuality (pp. 108-122). New York: Wiley.

Fava, M., Rankin, M. A., Alpert, J. E., Nierenberg, A. A., y Worthington, J. J. (1998). An open trial of oral sildenafil in antidepressant-induced sexual dysfunction. Psychotherapy and Psychosomatics, 67, 328-331. doi:10.1159/000012299.

Giargiari, T. D., Mahaffey, A. L., Craighead, W. E., y Hutchison, K. E. (2005). Appetitive responses to sexual stimuli are attenuated in individuals with low levels of sexual desire. Archives of Sexual Behavior, 34, 547-556.

Graham, C. A., Sanders, S. A., y Milhausen, R. R. (2006). The Sexual Excitation and Sexual Inhibition Inventory for Women: Psychometric properties. Archives of Sexual Behavior, 35, 397-410. doi:10.1007/ s10508-006-9041-7.

Graziottin, A., Serafini, A., y Palacios, S. (2009). Etiology, diagnostic algorithms and prognosis of female sexual dysfunction. Maturitas, 63, 128-134. doi:10.1016/j.maturitas.2009.04.007.

Hayes, R. D., Dennerstein, L., Bennett, C. M., y Fairley, C. K. (2008). What is the 'True' prevalence of female sexual dysfunctions and does the way we assess these conditions have an impact? Journal of Sexual Medicine, 5, 777-787. doi:10.1111/j.17436109.2007.00768.x.

Hicks, T., y Leitenberg, H. (2001). Sexual fantasies about one's partner someone else: Gender differences in incidence and frequency. Journal of Sex Research, 38, 43-50. doi:10.1080/00224490109552069.

Huck, S. W. (2000). Reading statistics and research (3rd ed.). New York: Addison Wesley Longman.

Impett, E., y Peplau, L. A. (2003). Sexual compliance: Gender, motivational, and relationship perspectives. Journal of Sex Research, 40, 87-100.

Janssen, E., y Bancroft, J. (2007). The dual control model: The role of sexual inhibition and excitation in sexual arousal and behavior. En E. Janssen (Ed.), The psychophysiology of sex (pp. 197-222). Bloomington: Indiana University Press.

Janssen, E., Vorst, H., Finn, P., y Bancroft, J. (2002a). The sexual inhibition (SIS) and sexual excitation (SES) scales: I. Measuring sexual inhibition and excitation proneness in men. The journal of Sex Research, 39, 114 126. doi:10.1080/00224490209552130.

Janssen, E., Vorst, H., Finn, P., y Bancroft, J. (2002b). The Sexual Inhibition (SIS) and sexual excitation (SES) scales: II. Predicting psychophysiological response patterns. The Journal of Sex Research, 39, 127-132. doi:10.1080/00224490209552131.

Jöreskog, K. G., y Sörbom, D. (1993). LISREL 8: Structural equation modeling with the SIMPLIS command language. Hillsdale, NJ: Erlbaum.

Laumann, E. O., Gagnon, J. H., Michael, R. T., y Michaels, S. (1994). The social organization of sexuality. Chicago: University of Chicago Press.

Levine, S. B. (2010). Hypoactive Sexual Desire Disorder in Men: Basic types, causes, and treatment. Psychiatric Times, 40-43.

McCabe, M. P. (2005). The role of performance anxiety in the development and maintenance of sexual dysfunction in men and women. International Journal of Stress Management, 12, 379-388. doi:10.1037/10725245.12.4.379.

Moyano, N., y Sierra, J. C. (2013). Relationships between personality traits and positive/negative sexual cognitions. International Journal of Clinical and Health Psychology, 13, 189-196. doi: 10.1016/S16972600(13)70023-1

Ortega, V., Zubeidat, I., y Sierra J. C. (2006). Further examination measurement properties of Spanish of the Sexual Desire Inventory with undergraduates and adolescent students. Psychological Reports, 99, 147-165. doi:10.2466/pr0.99.1.147-165.

Peplau, L. A. (2003). Human sexuality: How do men and women differ? Current Directions in Psychological Science, 12, 37-40.

Petersen, J., y Hyde, J. S. (2011). Gender differences in sexual attitudes and behaviors: A review of meta-analytic results and large datasets. Journal of Sex Research, 48, 149-165. doi:10.1080/00224499.2011.551851.

Prause, N., y Graham, C. A. (2007). Asexuality: Classification and characterization. Archives of Sexual Behavior, 36, 341-356. doi:10.1007/ s10508-006-9142-3.

Prause, N., Janssen, E., y Hetrick, W. P. (2008). Attention and emotional response to sexual stimuli and their relationship to sexual desire. Archives of Sexual Behavior, 37, 934-949. doi:10.1007/s10508-007-9236-6.

Ramiro, M. T., Teva, I., Bermúdez, M. P. y Buela-Casal, G. (2013). Social support, self-esteem and depression: Relationship with riks for sexually transmitted infections/HIV transmision. International Journal of Clinical and Health Psychology, 13, 181-188. doi: 10.1016/S16972600(13)70022-X

Rizvi, S. K., Yeung, N. W., y Kennedy, S. H. (2011). Instruments to measure sexual dysfunction in community and psychiatric populations. Journal of Psychosomatic Research, 70, 99-109. doi:10.1016/j. jpsychores.2010.05.009.

Rodríguez-Franco, L., López-Cepero Borrego, J., Rodríguez-Díaz, F.J., Bringas-Molleda, C., Estrada-Pineda, C., Antuña-Bellerín, M. A., y Quevedo-Blasco, R. (2012). Labeling dating abuse: Undetected abuse among Spanish adolescents and Young adults. International Journal of Clinical and Health Psychology, 12, 55-67.

Rosen, R., Brown, C., Heiman, J., Leiblum, S., Meston, C., Shabsigh, R.,... . D'Agostino, R. (2000). The Female Sexual Function Index (FSFI): A multidimensional self-report instrument for the assessment of female sexual function. Journal of Sex and Marital Therapy, 26, 191-208. doi:10.1080/009262300278597.

Salemink, E., y Van Lankveld, J. (2006). The effects of increasing neutral distraction on sexual responding of women with and without sexual problems. Archives of Sexual Behavior, 35, 179-190. doi:10.1007/ s10508-005-9014-2.

Santos-Iglesias, P., Calvillo, G., y Sierra, J. C. (2013). A further examination of Levine's model of sexual desire. Psychology \& Sexuality 4, 34-45

Santos-Iglesias, P., y Sierra, J. C. (2013). Predictors of sexual assertiveness: The role of sexual desire, arousal, attitudes, and partner abuse. Archives of Sexual Behavior, 42, 1043-1052. doi:10.1007/s10508-012-9998-3.

Sapien, J. S., y Córdoba, D. I. (2011). Comportamiento sexual de varones durante el embarazo: casos en la Ciudad de México. Terapia Psicológica, 29, 185-190. doi:10.4067/S0718-48082011000200005. 
Sarasúa, B., Zubizarreta, I., de Corral, P., y Echeburúa, E. (2012). Factores de vulnerabilidad y de protección del impacto emocional en mujeres adultas víctimas de agresiones sexuales. Terapia Psicológica, 30, 7-18. doi:10.4067/S0718-48082012000300002.

Schumacker, R. E., y Lomax, R. G. (2004). A beginner's guide to structural equation modeling. Mahwah, NJ: Lawrence ErlbaumAssociates.

Sierra, J. C., Perla, F., y Gutiérrez-Quintanilla, R. (2010). Actitud hacia la masturbación en adolescentes: propiedades psicométricas de la versión española del Attitudes Toward Masturbation Inventory. Universitas Psychologica, 9, 531-542.

Sierra, J. C., Vallejo-Medina, P., Santos-Iglesias, P., y Lameiras Fernández, M. (2012). Validación del Massachusetts General Hospital-Sexual Functioning Questionnaire (MGH-SFQ) en población española. Atención Primaria, 44, 516-526. doi:10.1016/j.aprim.2012.02.004.

Sims, K. E., y Meana, M. (2010). Why did passion wane? A qualitative study of married women's attributions for declines in sexual desire.Journal of Sex and Marital Therapy, 36, 360-380. doi:10.1080/0092623X.2010.498727.

Slob, A. K., Bax, C. M., Hop, W. C., Rowland, D. L., y van der Werfften Bosch, J. J. (1996). Sexual arousability and the menstrual cycle. Psychoneuroendocrinology, 21, 545-558. doi:10.1016/0306-4530(95)00058-5.

Spector, I. P., Carey, M. P., y Steinberg, L. (1996). The Sexual Desire Inventory: Development, factor structure and evidence of reliability. Journal of Sex and Marital Therapy, 22, 175-190.
Sprecher, S., y Regan, P. C. (1996). College virgins: How men and women perceive their sexual status. Journal of Sex Research, 33, 3-15. doi:10.1080/00224499609551810.

Taylor, J. F., Rosen, R. C., y Leiblum, S. R. (1994). Self- report assessment of female sexual function: Psychometric evaluation of the Brief Index of Sexual Functioning for women. Archives of Sexual Behavior, 23 , 627-643.

Tiefer, L. (2001). General discussion. En J. Bancroft (Ed.), The role of theory in sex research (pp. 235-236). Bloomington: Indiana University Press.

Van Anders, S. (2012). Testosterone and sexual desire in healthy women and men. Archives of Sexual Behavior, 41, 1471-1484. doi:10.1007/ s10508-012-9946-2.

Van Lankveld, J., y Bergh, S. (2008). The interaction of state and trait aspects of self-focused attention affects genital, but not subjective, sexual arousal in sexually functional women. Behavior Research and Therapy, 46, 514-528.

Winters, J., Christoff, K., y Gorzalka, B. B. (2008). Conscious regulation of sexual arousal in men. Journal of Sex Research, 46, 330-343. doi:10.1080/00224490902754103.

Wylie, K., y Mimoun, S. (2009). Sexual response models in women. Maturitas, 63, 112-115. doi:10.1016/j.maturitas.2009.03.007. 
